\title{
Core Stability of Vertex Cover Games
}

\author{
Qizhi Fang, Liang Kong, and Jia Zhao
}

Abstract. In this paper, we focus on the core stability of vertex cover games, which arise from vertex cover problems on graphs. Based on duality theory of linear programming, we prove that a balanced vertex cover game has a stable core if and only if every edge belongs to a maximum matching in the underlying graph. We also prove that for a totally balanced vertex cover game, the core largeness, extendability, and exactness are all equivalent, which implies core stability. Furthermore, we show that core stability and the three related properties can be determined efficiently.

\section{Introduction}

A transferable cooperative game $\Gamma=(N, \gamma)$ consists of a set of players $N=$ $\{1,2, \ldots, n\}$ and a characteristic function $\gamma: 2^{N} \rightarrow R$, where for each subset $S \subseteq N$ of players, $\gamma(S)$ represents the revenue or cost achieved by the players in $S$ without assistance of other players. One of the scopes of cooperative game theory is to study how to distribute the total revenue or cost $\gamma(N)$ among the participants in a fair way. Different philosophies result in different solution concepts, such as core, Shapley value, nucleolus, and stable set. The concept of a stable set was first introduced in [von Neumann and Morgenstern 44], in which the authors claimed that it is very useful in the analysis of many bargaining situations.

While the core and the stable set are different, it was shown in [Shapley 71] that for convex games, the core is the unique stable set. This result motivated researchers to study the following problem: when do the core and the stable set

(C) A K Peters, Ltd.

|542-795|/08 \$0.50 per page 
coincide, that is, when is the core stable? However, it is difficult to investigate the existence and properties of the stable set because of the complexity of its definition. Recently, it was shown in [Jain and Vohra 06] that determining the existence of a stable set and checking the core stability for general cooperative games is decidable. However, it is unlikely that the algorithms presented by Jain and Vohra will be efficient. As far as the core stability for concrete cooperative game model is concerned, only a few results have been obtained. The core stability for assignment games is studied in [Solymosi and Raghavan 01], and it is studied in [Bietenhader and Okamoto 04] for minimum coloring games defined on perfect graphs.

In this paper, we focus on core stability of vertex cover games introduced in [Deng et al. 99], which arise from cost allocation problems related to vertex covers on graphs. We show that a vertex cover game has a stable core if and only if every edge belongs to a maximum matching in the corresponding graph. We also discuss three related properties: core largeness, extendability, and exactness of the game. We prove that all these properties are equivalent for vertex cover games defined on bipartite graphs, and furthermore, are equivalent to a graphtheoretic property that every matching is contained in a maximum matching. We also show how to test this equivalent condition in polynomial time.

\section{Definitions and Preliminaries}

We begin with some concepts and notions from graph theory and cooperative game theory.

\subsection{Graphs}

Let $G=(V, E)$ be an undirected graph with vertex set $V$ and edge set $E$. The neighborhood $N_{G}(v)$ of vertex $v \in V$ is the set of vertices adjacent to $v$ in $G$. For $v \in V$, denote by $E(v)$ the set of edges incident to $v$, and by $G \backslash v$ the subgraph obtained from $G$ by deleting the vertex $v$ together with its incident edges.

A subset $K \subseteq V$ is called a vertex cover of $G$ if each edge of $G$ has at least one end in $K$. A vertex cover is minimum if it has the minimum cardinality among all the vertex covers of $G$. The vertex cover number, denoted by $\tau(G)$, is defined as the cardinality of any minimum vertex cover of $G$.

A subset $M \subseteq E$ is called a matching of $G$ if no two edges in $M$ have an end in common. A matching of $G$ is called maximal if adding any other edge to it makes it no longer a matching, and called maximum if it has the maximum cardinality among all the matchings. The matching number, denoted by $\nu(G)$, equals the cardinality of any maximum matching of $G$. 
In general, $\nu(G) \leq \tau(G)$, and equality does not hold. However, if $G$ is a bipartite graph, we do have

$$
\nu(G)=\tau(G) .
$$

This result is called König's theorem [Reinhard 00].

\subsection{Core Stability}

Throughout this paper, all the games concerned are cost games, that is, the values of the characteristic function specify the costs of all coalitions. Let $\Gamma=(N, \gamma)$ be a cooperative game. A vector $x=\left(x_{1}, x_{2}, \ldots, x_{n}\right)$ is called an imputation if $\sum_{i \in N} x_{i}=\gamma(N)$ and for all $i \in N$, we have $x_{i} \leq \gamma(\{i\})$. The set of imputations is denoted by $I(\Gamma)$. The core is defined as

$$
C(\Gamma)=\left\{x \in R^{n}: x(N)=\gamma(N) \text { and } x(S) \leq \gamma(S), \text { for all } S \subseteq N\right\},
$$

where $x(S)=\sum_{i \in S} x_{i}$. The game $\Gamma=(N, v)$ is balanced if $C(\Gamma)$ is nonempty, and totally balanced if every subgame (i.e., the game obtained by restricting the player set to a coalition and the characteristic function to the power set of that coalition) is balanced.

The concept of stability goes back to [von Neumann and Morgenstern 44]. Given two imputations $x, y \in I(\Gamma)$, we say that $x$ dominates $y$ if there is a nonempty coalition $S$ such that $x(S) \geq \gamma(S)$ and for all $i \in S, x_{i}<y_{i}$. A set $\mathcal{F} \subseteq I(\Gamma)$ is stable if any two imputations in $\mathcal{F}$ do not dominate each other and any imputation not in $\mathcal{F}$ can be dominated by an imputation in $\mathcal{F}$. Since the core allocations do not dominate each other, to say that the core is stable simply means that any imputation not in the core can be dominated by a core imputation. Formally, the core of a balanced game $\Gamma$ is stable if for every $y \in$ $I(\Gamma) \backslash C(\Gamma)$, there exist an $x \in C(\Gamma)$ and a nonempty coalition $S \subset N$ such that $x(S)=\gamma(S)$ and $x_{i}<y_{i}$ for all $i \in S$.

\subsection{Vertex Cover Game}

Now let us give the definition of vertex cover games. Given an undirected graph $G=(V, E)$, the corresponding vertex cover game $\Gamma_{G}=(E, \gamma)$ is defined as follows:

1. The player set is $E=\left\{e_{1}, e_{2}, \ldots, e_{n}\right\}$.

2. For each coalition $S \subseteq E, \gamma(S)$ is the cardinality of a minimum vertex cover in the induced subgraph $G[S]=(V, S)$.

Vertex cover games fall into the scope of the class of combinatorial cooperative games studied in [Deng et al. 99]. With the duality theory of linear programming, 
its authors presented a necessary and sufficient condition for a vertex cover game to be balanced.

Theorem 2.I. [Deng et al. 99] Given a graph $G=(V, E)$, the vertex cover game defined on $G$ is balanced if and only if $\nu(G)=\tau(G)$. In such case, an imputation is in the core if and only if it is a convex combination of the indicator vectors of maximum matchings of $G$.

Since $\nu(G)=\tau(G)$ for a bipartite graph $G$, it follows from Theorem 2.1 that the corresponding vertex cover game is balanced. Furthermore, [Deng et al. 00] gives a necessary and sufficient condition for a vertex cover game to be totally balanced.

Theorem 2.2. [Deng et al. 00] Let $\Gamma_{G}=(E, \gamma)$ be the vertex cover game defined on the graph $G=(V, E)$. Then $\Gamma_{G}$ is totally balanced if and only if $G$ is a bipartite graph.

\section{Vertex Cover Game with Stable Core}

Let $\Gamma_{G}=(E, \gamma)$ be the vertex cover game defined on the graph $G=(V, E)$. Before discussing the core stability of $\Gamma_{G}$, we need some lemmas.

Lemma 3.I. [Deng et al. 99] We have $x \in C\left(\Gamma_{G}\right)$ if and only if

(1) $x \geq 0$;

(2) $x(E)=\gamma(E)$;

(3) $x(E(v)) \leq 1$ for each $v \in V$.

Lemma 3.2. Let the vertex cover game $\Gamma_{G}=(E, \gamma)$ be balanced. If $\Gamma_{G}$ has a stable core, then there exists a core allocation $x$ such that $x(e)>0$ for all $e \in E$.

Proof. Suppose that there is an edge $e_{0} \in E$ such that $x\left(e_{0}\right)=0$ for all $x \in C\left(\Gamma_{G}\right)$. Since $\gamma(E)>0$, there exist $e_{1} \in E$ and $\hat{x} \in C\left(\Gamma_{G}\right)$ such that $\hat{x}\left(e_{1}\right)>0$. Construct a vector $y: E \rightarrow R^{+}$based on $\hat{x}$ as follows:

$$
y(e)= \begin{cases}\hat{x}(e) & \text { if } e \notin\left\{e_{0}, e_{1}\right\}, \\ \hat{x}\left(e_{1}\right) & \text { if } e=e_{0}, \\ 0 & \text { if } e=e_{1} .\end{cases}
$$


Obviously, $y \in I\left(\Gamma_{G}\right) \backslash C\left(\Gamma_{G}\right)$. Since $C\left(\Gamma_{G}\right)$ is stable, there exist $x^{*} \in C\left(\Gamma_{G}\right)$ and a nonempty set $S \subseteq E$ such that $x^{*}$ dominates $y$ on $S$.

Note that it must be the case that $e_{0} \in S$. Otherwise, we distinguish two cases:

(a) $e_{1} \in S$. Since $y\left(e_{1}\right)=0$, each core allocation cannot dominate $y$ on $S$.

(b) $e_{1} \notin S$. Since $\hat{x} \in C\left(\Gamma_{G}\right)$ and $y(e)=\hat{x}(e)$ for each $e \in S, x^{*}$ cannot dominate $y$ on $S$.

Therefore, we have $\gamma(S)=x^{*}(S)=x^{*}\left(S \backslash\left\{e_{0}\right\}\right)<y\left(S \backslash\left\{e_{0}\right\}\right)=\hat{x}\left(S \backslash\left\{e_{0}\right\}\right) \leq$ $\gamma\left(S \backslash\left\{e_{0}\right\}\right) \leq \gamma(S)$, which is a contradiction.

The following theorem is the main result of this section.

Theorem 3.3. Let $\Gamma_{G}=(E, \gamma)$ be a balanced vertex cover game defined on the graph $G=(V, E)$. Then $\Gamma_{G}$ has a stable core if and only if each edge $e \in E$ belongs to a maximum matching of $G$.

Proof. Necessity. Assume that $\Gamma_{G}=(E, \gamma)$ has a stable core. By Lemma 3.2, for each $e \in E$, there exists a core allocation $x$ such that $x(e)>0$. It also follows from Theorem 2.1 that $x$ is a convex combination of the indicator vectors of maximum matchings. Hence $e$ belongs to at least one maximum matching of $G$.

Sufficiency. Given $y \in I\left(\Gamma_{G}\right) \backslash C\left(\Gamma_{G}\right)$, according to Lemma 3.1, there is a vertex $v \in V$ such that $y(E(v))>1$. Let $S=E(v) \backslash\{e \in E(v): y(e) \leq 0\}$, which is denoted by $S=\left\{e_{1}, e_{2}, \ldots, e_{k}\right\}$. It is easy to see that $S \neq \varnothing, y(S)>1$, and $y\left(e_{i}\right)>0, i=1,2, \ldots, k$. By our assumption, each $e_{i} \in S$ belongs to a maximum matching of $G$, namely $M_{i}, i=1,2, \ldots, k$. Since $S \subseteq E(v)$ and $M_{i}$ is a matching, $M_{i} \cap S$ contains the unique element $e_{i}$ in $E(v), i=1,2, \ldots, k$.

We denote by $\mathbb{I}_{1}, \mathbb{I}_{2}, \ldots, \mathbb{I}_{k}$ the respective indicator vectors of $M_{1}, M_{2}, \ldots, M_{k}$. Define $x \in R^{|E|}$ as follows:

$$
x=\lambda_{1} \mathbb{I}_{1}+\lambda_{2} \mathbb{I}_{2}+\cdots+\lambda_{k} \mathbb{I}_{k},
$$

where $\lambda_{i}=\frac{y\left(e_{i}\right)}{y(S)}, i=1,2, \ldots, k$. Obviously, $\lambda_{i}>0$ and $\sum_{i=1}^{k} \lambda_{i}=1$. From Theorem 2.1, we conclude that $x \in C\left(\Gamma_{G}\right)$ and

$$
\begin{aligned}
& x(S)=\sum_{i=1}^{k} \lambda_{i} \mathbb{I}_{i}(S)=\sum_{i=1}^{k} \lambda_{i}\left|M_{i} \cap S\right|=1=\gamma(S), \\
& x\left(e_{i}\right)=\lambda_{i}=\frac{y\left(e_{i}\right)}{y(S)}<y\left(e_{i}\right), \text { for all } e_{i} \in S .
\end{aligned}
$$

That is, $x$ dominates $y$ on $S$. Therefore, $C\left(\Gamma_{G}\right)$ is stable. 
Now we discuss the algorithm for checking whether a vertex cover game possesses a stable core. The problem is stated as follows:

Problem A: Check core stability of vertex cover game.

Instance: The vertex cover game $\Gamma_{G}$ defined on a graph $G$.

Question: Does $\Gamma_{G}$ possess a stable core?

In [Deng et al. 99], it was shown that testing core nonemptiness of a vertex cover game can be done in polynomial time. Theorem 3.3 yields that Problem $\mathrm{A}$ is equivalent to the problem of checking whether every edge belongs to a maximum matching of $G$. This can be done as follows:

First find a maximum matching $M$ and let $E^{*}=E \backslash M$. For each $e=(u, v) \in$ $E^{*}$, we replace $e$ by two new edges $\left(u, u^{\prime}\right)$ and $\left(v, v^{\prime}\right)$ (where $u^{\prime}$ and $v^{\prime}$ are two new pendant vertices), and check whether there is an $M$-augmenting path $P$ with one endpoint being $u^{\prime}$ or $v^{\prime}$. If such an augmenting path $P$ exists, then we modify $E^{*}:=E^{*} \backslash(P \cup\{e\})$; otherwise, $e$ is picked out as a "bad edge." Repeat this procedure until $E^{*}=\varnothing$ or a "bad edge" occurs. Obviously, an edge belongs to a maximum matching of $G$ if and only if it is not a "bad edge." Therefore, we have the following result.

Theorem 3.4. The problem of checking core stability of a vertex cover game can be solved in polynomial time.

\section{Exactness, Extendability, and Core Largeness}

In this section, we discuss three properties closely related to core stability: core largeness, game exactness, and extendability. Given a cooperative game $\Gamma=$ $(N, \gamma)$ with $|N|=n, \Gamma$ is said to have a large core if for every $y \in R^{n}$ satisfying $y(S) \leq \gamma(S)$ for all $S \subseteq N$, there exists $x \in C(\Gamma)$ such that $x \geq y$. The game $\Gamma$ is called extendable if for every nonempty set $S \subset N$ and every core allocation $y$ of the subgame $\left(S, \gamma_{S}\right)$, there exists $x \in C(\Gamma)$ such that $x_{i}=y_{i}$ for all $i \in S$. Finally, $\Gamma$ is called exact if for each $S \subset N$ there exists $x \in C(\Gamma)$ such that $x(S)=\gamma(S)$.

It was shown in [Kikuta and Shapley 86] that if a balanced game has a large core, then it is extendable; and if a balanced game is extendable, then it has a stable core. It is shown in [Sharkey 82] that if a totally balanced game has a large core, then it must be exact. We summarize these results in the following theorem. 
Theorem 4.I. [Kikuta and Shapley 86, Sharkey 82] Let $\Gamma=(N, \gamma)$ be a totally balanced game. Then

(1) $\Gamma$ has a large core implies that $\Gamma$ is extendable;

(2) $\Gamma$ is extendable implies that $\Gamma$ is exact and has a stable core.

Theorem 2.2 tells us that $\Gamma_{G}$ is totally balanced if and only if $G$ is a bipartite graph. Therefore, in the following we focus on bipartite graphs. We shall prove that the three properties exactness, extendability, and core largeness are equivalent for vertex cover games defined on bipartite graphs.

Theorem 4.2. Let $\Gamma_{H}=(E, \gamma)$ be the vertex cover game defined on the bipartite graph $H=\left(V_{1}, V_{2} ; E\right)$. Then the following conditions are equivalent:

(1) $C\left(\Gamma_{H}\right)$ is large;

(2) $\Gamma_{H}$ is extendable;

(3) $\Gamma_{H}$ is exact;

(4) every matching is contained in a maximum matching of $H$.

By Theorem 4.1, we have (1) $\Rightarrow(2) \Rightarrow(3)$. It remains to prove $(3) \Rightarrow(4)$ and (4) $\Rightarrow(1)$.

Proof of $(3) \Rightarrow(4)$. Suppose that $\Gamma_{H}=(E, \gamma)$ is exact. Let $M^{*}$ be a matching of $H$. By the definition of exactness, there is $x^{*} \in C\left(\Gamma_{H}\right)$ such that $x^{*}\left(M^{*}\right)=$ $\gamma\left(M^{*}\right)=\left|M^{*}\right|$. Let $\mathcal{M}=\left\{M_{1}, M_{2}, \ldots, M_{k}\right\}$ be the set of maximum matchings of $H$. It follows from Theorem 2.1 that $x^{*}$ can be expressed as $x^{*}=\sum_{i=1}^{k} \lambda_{i} \mathbb{I}_{i}$, where $\mathbb{I}_{i}$ is the indicator vector of the matching $M_{i}, \lambda_{i} \geq 0(i=1,2, \ldots, k)$, and $\sum_{i=1}^{k} \lambda_{i}=1$. Then we have

$$
x^{*}\left(M^{*}\right)=\sum_{i=1}^{k} \lambda_{i} \mathbb{I}_{i}\left(M^{*}\right)=\sum_{i=1}^{k} \lambda_{i}\left|M^{*} \cap M_{i}\right| \leq \sum_{i=1}^{k} \lambda_{i}\left|M^{*}\right|=\left|M^{*}\right| .
$$

Therefore, equality holds throughout in the above formula, which implies that $M^{*} \cap M_{i}=M^{*}$ for any $M_{i}$ with $\lambda_{i}>0$. That is, $M^{*}$ is contained in at least one maximum matching of $H$. 
To prove $(4) \Rightarrow(1)$, we need some additional facts. The first is found in [Van Gellekom et al. 99]. For a game $\Gamma=(N, \gamma)$ with $|N|=n$, the set of lower vectors is defined as

$$
L(\Gamma)=\left\{y \in R^{n}: y(S) \leq \gamma(S), \text { for all } S \subseteq N\right\}
$$

Lemma 4.3. [Van Gellekom et al. 99] Let $\Gamma=(N, \gamma)$ be a balanced game. Then $\Gamma=(N, \gamma)$ has a large core if and only if $y(N) \geq \gamma(N)$ for each extreme point $y$ of $L(\Gamma)$.

In order to establish the core largeness of $\Gamma_{H}$, we need to characterize the extreme points of $L\left(\Gamma_{H}\right)$. For this purpose, we give an alternative description of $L\left(\Gamma_{H}\right)$. Let $\mathcal{S}=\left\{T: T \subseteq E(v), v \in V_{1} \cup V_{2}\right\}$, and

$$
L^{\prime}\left(\Gamma_{H}\right)=\left\{y \in R^{n}: y(S) \leq 1 \text { for all } S \in \mathcal{S}\right\} .
$$

Lemma 4.4. Let $\Gamma_{H}=(E, \gamma)$ be the vertex cover game defined on a bipartite graph $H=\left(V_{1}, V_{2} ; E\right)$. Then

(1) $L\left(\Gamma_{H}\right)=L^{\prime}\left(\Gamma_{H}\right)$.

(2) Each extreme point of $L^{\prime}\left(\Gamma_{H}\right)$ is the indicator vector of a maximal matching of $H$.

Proof. (1) It is easy to see that $L\left(\Gamma_{H}\right) \subseteq L^{\prime}\left(\Gamma_{H}\right)$. Then let us prove the other direction. Given $y \in L^{\prime}\left(\Gamma_{H}\right)$, we have to check that $y(S) \leq \gamma(S)$ for every $S \subseteq E$. Assume that $\gamma(S)=k$. Then $S$ can be divided into $k$ disjoint subsets, namely $S_{1}, S_{2}, \ldots, S_{k}$, where $S_{i} \in \mathcal{S}, i=1,2, \ldots, k$. Since $y \in L^{\prime}\left(\Gamma_{H}\right), y\left(S_{i}\right) \leq 1$ for each $i=1,2, \ldots, k$. Therefore, $y(S)=\sum_{i=1}^{k} y\left(S_{i}\right) \leq k=\gamma(S)$. That is, $y \in L\left(\Gamma_{H}\right)$. Hence $L\left(\Gamma_{H}\right)=L^{\prime}\left(\Gamma_{H}\right)$.

(2) First, we show that the extreme points of $L^{\prime}\left(\Gamma_{H}\right)$ are nonnegative. Suppose that $y$ is an extreme point of $L^{\prime}\left(\Gamma_{H}\right)$ with at least one negative component. Then we define two vectors $y^{1}$ and $y^{2}$ as follows:

$$
y_{i}^{1}=\left\{\begin{array}{ll}
y_{i} & \text { if } y_{i} \geq 0, \\
0 & \text { if } y_{i}<0,
\end{array} \quad y_{i}^{2}= \begin{cases}y_{i} & \text { if } y_{i} \geq 0 \\
2 y_{i} & \text { if } y_{i}<0\end{cases}\right.
$$

It is easy to see that $y^{1}, y^{2} \in L^{\prime}\left(\Gamma_{H}\right)$. Since $y \neq y^{1}, y \neq y^{2}$, and $y=\frac{y^{1}+y^{2}}{2}$, it follows that $y$ is not an extreme point of $L^{\prime}\left(\Gamma_{H}\right)$.

Second, we show the correctness of statement (2) of this lemma. Consider the following polyhedron:

$$
\begin{aligned}
L^{\prime \prime}\left(\Gamma_{H}\right) & =\left\{y \in R^{n}: y(S) \leq 1, \forall S \in \mathcal{S} ; y \geq 0\right\} \\
& =\left\{y \in R^{n}: y(E(v)) \leq 1, \forall v \in V ; y \geq 0\right\},
\end{aligned}
$$


where the second equality follows from the definition of $\mathcal{S}$. Since the extreme points of $L^{\prime}\left(\Gamma_{H}\right)$ are nonnegative, each extreme point of $L^{\prime}\left(\Gamma_{H}\right)$ is also an extreme point of $L^{\prime \prime}\left(\Gamma_{H}\right)$. Let $y^{*}$ be an extreme point of $L^{\prime \prime}\left(\Gamma_{H}\right)$. Based on linear programming theory, there exists a nonnegative vector $\omega$ such that $y^{*}$ is the unique optimal solution of the following linear program:

$$
\mathrm{LP}^{*}: \quad \max \left\{\omega^{t} y: y(E(v)) \leq 1, \forall v \in V ; y \geq 0\right\} .
$$

Since $H$ is a bipartite graph, the coefficient matrix of the constraints of $\mathrm{LP}^{*}$ is totally balanced. Hence, $y^{*}$ must be an integer vector, i.e., a $\{0,1\}$-vector, that is the indicator vector of a matching $M$ of $H$.

Assume that $M$ is not a maximal matching. Then there exists a matching $M^{\prime}$ with $M \subset M^{\prime}$. It follows that the indicator vector of $M^{\prime}$ is also an optimal solution of $\mathrm{LP}^{*}$, contradicting the fact that $y^{*}$ is the unique optimal solution. Therefore, $M$ is a maximal matching.

With Lemmas 4.3 and 4.4 , we are ready to prove $(4) \Rightarrow(1)$.

Proof of $(4) \Rightarrow(1)$. Let $H=\left(V_{1}, V_{2} ; E\right)$ satisfy condition (4), and let $y$ be an extreme point of $L\left(\Gamma_{H}\right)$. It follows from Lemma 4.4 that $y$ is the indicator vector of a maximal matching $M$. By condition (4), $M$ is certainly a maximum matching of $H$. Hence $y(E)=|M|=\gamma(E)$. By Lemma 4.3, $\Gamma_{H}$ has a large core.

There is also an algorithmic problem:

Problem B: Check extendability, exactness, and core largeness of a vertex cover game on a bipartite graph.

Instance: The vertex cover game $\Gamma_{H}$ defined on a bipartite graph $H$. Question: Is $\Gamma_{H}$ extendable, exact, and with large core?

Thanks to Theorem 4.2, Problem B is equivalent to determining whether every matching is contained in a maximum matching of $H$. In order to investigate the algorithm for this problem, we need some preparation.

We say that a graph $G=(V, E)$ is matching-normal if every maximal matching of $G$ is already a maximum matching of $G$. It is easy to see that $G$ being matching-normal is equivalent to condition (4) of Theorem 4.2. Note that finding a maximal matching with minimum cardinality (called a minimum maximal matching) is NP-hard, even for planar or bipartite graphs [Yannakakis and Gavril 80]. However, in the following we shall show that checking whether a bipartite graph $G$ is matching-normal, i.e., checking whether the cardinality of a minimum maximal matching of $G$ equals the matching number $\nu(G)$, is polynomially solvable. 
A vertex $v$ of a graph $G=(V, E)$ is critical if it satisfies $(1) \nu(G \backslash v)=\nu(G)-1$ and (2) there is a maximum matching of the subgraph $G \backslash v$ saturating all the vertices in $N_{G}(v)$.

Note that the maximum matching problem in a bipartite graph can be transformed into the max-flow problem in the associated network, whereby each vertex of the original graph is connected to the "source" or "sink" by a related arc $[\mathrm{Pa}-$ padimitriou and Steiglitz 82, Chapter 10]. For a bipartite graph $H=\left(V_{1}, V_{2} ; E\right)$, we can identify its critical vertices as follows:

Taking a vertex $v \in V_{1} \cup V_{2}$, we first find a maximum matching $M_{v}$ in $H \backslash v$. If $\left|M_{v}\right|=\nu(H)-1$, then instead of finding a maximum matching in $H \backslash v$ saturating all the vertices in $N_{H}(v)$, one can find a max-flow in the associated network with the lower bounds of capacities being 1 on the related arcs of $N_{H}(v)$. Therefore, we have the following result.

Lemma 4.5. Given a bipartite graph $H=\left(V_{1}, V_{2} ; E\right)$, the critical vertices of $H$ can be identified in polynomial time.

Now we are in a position to characterize a matching-normal bipartite graph.

Lemma 4.6. Let $H=\left(V_{1}, V_{2} ; E\right)$ be a bipartite graph. Then $H$ is matching-normal if and only if there is no critical vertex in $H$.

Proof. Necessity. If there is a critical vertex $v \in V_{1} \cup V_{2}$, then there must be a maximum matching $M^{\prime}$ of the subgraph $H \backslash v$ such that $\left|M^{\prime}\right|=\nu(H)-1$ and $M^{\prime}$ saturates all the vertices in $N_{G}(v)$. It is easy to see that $M^{\prime}$ is in fact a maximal matching in the original graph $H$, implying that $H$ is not matching-normal.

Sufficiency. Suppose that $H$ is not matching-normal and $M^{\prime}$ is a maximal matching of $H$ with $\left|M^{\prime}\right|<\nu(H)$. Choose a maximum matching $M^{*}$ of $H$ such that $\left|M^{\prime} \cap M^{*}\right|$ is as large as possible.

Consider $F=G\left[M^{\prime} \triangle M^{*}\right]$, where $M^{\prime} \triangle M^{*}$ denotes the symmetric difference of $M^{\prime}$ and $M^{*}$. The choice of $M^{*}$ ensures that each component of $F$ is a path starting and ending with edges in $M^{*}$, and with edges alternately in $M^{\prime}$ and $M^{*}$. Select one component of $F$, denoted by $P_{0}$. Obviously, both end vertices of $P_{0}$ are $M^{*}$-saturated but $M^{\prime}$-unsaturated. According to König's theorem and its proof [Reinhard 00], we have the following claim.

Claim 4.7. There is a minimum vertex cover $K^{*}$ of $H$ containing at least one of the two end vertices of $P_{0}$, denoted by $v_{0}$. Moreover, $K^{*} \backslash v_{0}$ is a vertex cover of the subgraph $H \backslash v_{0}$. 
Thus we can obtain another matching $\widetilde{M}$ of $H$ by augmenting $M^{\prime}$ along all the components of $F$ except for $P_{0}$. It is easy to see the following facts:

(1) $|\widetilde{M}|=\left|M^{*}\right|-1=\nu(H)-1$;

(2) $\widetilde{M}$ is also a maximal matching of $H$, since all the $M^{\prime}$-saturated vertices are still saturated by $\widetilde{M}$;

(3) $\widetilde{M}$ is a maximum matching of the subgraph $H \backslash v_{0}$, which follows from Claim 4.7 and König's theorem.

Facts (1), (2), and (3) yield directly that $v_{0}$ is a critical vertex of $H$. This completes the proof.

With Theorem 4.2 and Lemmas 4.5 and 4.6, we have obtained the following result.

Theorem 4.8. The problem of checking extendability, exactness, and core largeness of the vertex cover game on a bipartite graph can be solved in polynomial time.

Acknowledgments. This work is supported by NCET (No.05-0598) and NSFC (No.10771200).

\section{References}

[Bietenhader and Okamoto 04] T. Bietenhader and Y. Okamoto. "Core Stability of Minimum Coloring Games." In Graph-Theoretic Concepts in Computer Science: 30th International Workshop, WG 2004, Bad Honnef, Germany, June 21-23, 2004, Revised Papers, Lecture Notes Computer Science 3353, pp. 389-401. Berlin: Springer, 2004 .

[Deng et al. 99] X. Deng, T. Ibaraki, and H. Nagamochi. "Algorithmic Aspects of the Core of Combinatorial Optimization Games." Mathematics of Operations Research 24 (1999), 751-766.

[Deng et al. 00] X. Deng, T. Ibaraki, H. Nagamochi, and W. Zang, "Totally Balanced Combinatorial Optimization Games." Mathematical Programming (Ser. A) 87 (2000), 441C452.

[Jain and Vohra 06] K. Jain and R. V. Vohra. "On Stability of the Core." Preprint, 2006. (Available at http://www.kellogg.northwestern.edu/faculty/vohra/ $\mathrm{ftp} /$ newcore.pdf.)

[Kikuta and Shapley 86] K. Kikuta and L. S. Shapley. "Core Stability in $n$-Person Games." Unpublished manuscript, 1986.

[Papadimitriou and Steiglitz 82] C. H. Papadimitriou and K. Steiglitz. Combinatorial Optimization: Algorithms and Complexity. Englewood Cliffs, NJ: Prentice-Hall, 1982. 
[Reinhard 00] D. Reinhard. Graph Theory, second edition. New York: Springer, 2000.

[Shapley 71] L. S. Shapley. "Cores and Convex Games." International Journal of Game Theory 1 (1971), 11-26.

[Sharkey 82] W. W. Sharkey. "Cooperative Games with Large Cores." International Journal of Game Theory 11 (1982), 175-182.

[Solymosi and Raghavan 01] T. Solymosi and T. E. S Raghavan. "Assignment Games with Stable Cores." International Journal of Game Theory 30 (2001), 177-185.

[Van Gellekom et al. 99] J. R. G. van Gellekom, J. A. M. Potters, and J. H. Reijnierse. "Prosperity Properties of TU-games." International Journal of Game Theory 28 (1999), 211-277.

[von Neumann and Morgenstern 44] J. von Neumann and O. Morgenstern. Theory of Games and Economic Behaviour. Princeton: Princeton University Press, 1944.

[Yannakakis and Gavril 80] M. Yannakakis and F. Gavril. "Edge Dominating Sets in Graphs." SIAM J. Appl. Math. 38 (1980), 363-372.

Qizhi Fang, Department of Mathematics, Ocean University of China, Qingdao 266071, P. R. China (qfang@ouc.edu.cn)

Liang Kong, Department of Mathematics and Physics, Hebei Normal University of Science and Technology, Qinhuangdao 066004, P. R. China (kongliangouc@yahho.com.cn)

Jia Zhao, Department of Mathematics, Ocean University of China, Qingdao 266071, P. R. China (gauss_newton@126.com)

Received March 25, 2008; accepted August 12, 2009. 